\title{
Ankara City Hospital COVID-19 Severity Score (ACCSES):A Calculation Tool in the Prediction of Severe Illness Requiring Intensive Care Follow-up
}

\section{Rahmet Guner}

Bircan Kayaaslan ( $\nabla$ drbican@gmail.com )

Imran Hasanoglu

Adalet Aypak

Hurrem Bodur

Ihsan Ates

Esragul Akinci

Deniz Erdem

Fatma Eser

Seval Izdes

Ayse Kaya Kalem

Aliye Bastug

Aysegul Karalezli

Aziz Surel

Muge Ayhan

Selma Karaahmetoglu

Isil Ozkocak

Emine Arguder

Burcu Ozdemir

Mehmet Nevzat Mutlu

Yesim Aybar Bilir

Elif Mukime Saricaoglu

Derya Gokcinar

Sibel Gunay

Bedia Dinc

Emin Gemcioglu

Ruveyda Bilmez

Omer Aydos

Dilek Asilturk

Osman Inan

Turan Buzgan 


\section{Research Article}

Keywords: COVID-19, SARS-CoV-2, Calculation Tool, Severity Score, Predictive Factors

Posted Date: August 25th, 2020

DOI: https://doi.org/10.21203/rs.3.rs-63960/v1

License: (c) (i) This work is licensed under a Creative Commons Attribution 4.0 International License. Read Full License 


\section{Abstract}

Aim: The aim of this study is to develop and explain an easy-to-use severity score calculation tool to predict severe COVID-19 cases that would need intensive care unit (ICU) follow-up.

Material method: The study was carried out in patients with laboratory-confirmed COVID-19 hospitalized in Ankara City Hospital between March 15, 2020, and June 15, 2020. The outcome was severe illness that required ICU follow-up. Univariate and binary logistic regression were used to create a prediction model by using potential predictive parameters obtained on the day of hospitalization. Youden's $J$ index was calculated with receiver-operator characteristic curves analysis in order to evaluate cut-off points, and predicted probability was calculated. The accuracy of the prediction model was tested by calculating the area under curve (AUC).

Results: Of the total of 1022 patients, 152 had a severe illness and required ICU follow-up. Among 68 variables, 20 parameters met the potential predictive factor condition for severe illness and were included in the development process for ANKARA CITY HOSPITAL COVID-19 SEVERITY SCORE (ACCSES). The ACCSES calculation tool was created by the final 9 parameters (sex, oxygen saturation, hemoglobin, platelet count, glomerular filtration rate, aspartate transaminase, procalcitonin, ferritin, and D-dimer). AUC was $0.96(95 \% \mathrm{Cl}, 0.95-0.98)$.

Conclusion: We developed a simple, accessible, easy to use calculation tool, ACCSES, with good distinctive power for a severe illness that required ICU follow-up. The clinician can easily predict the course of COVID-19 and decide the procedure and facility of further follow-up simply using available clinical and laboratory values of patients upon admission.

\section{Introduction}

The world has been under threat of the novel coronavirus disease (COVID-19) since the last days of 2019. Up to 14 June 2020, a total of 12.015 .193 confirmed cases, resulting in 549.247 deaths have been reported [1]. Although the disease has a wide clinical spectrum from asymptomatic infection to critically ill [2], a small number of COVID-19 patients experience severe illness that can result in death. The China Center for Disease Control and Prevention reported mild disease, serious illness and critical illness as $81 \%, 14 \%$ and $5 \%$ in 44.672 confirmed cases, respectively [3]. The case fatality rate was reported as $2.3 \%$, but had increased to $49.0 \%$ in critical cases.

Since there is no specific treatment and the current vaccine for the new coronavirus (severe acute respiratory syndrome coronavirus-2 [SARS-CoV-2]) so far, the early recognition of patients who will worsen and the provision of aggressive supportive treatment is the essential point of patient management. Early detection of patients whose illness will progress helps the physician to decide whether the patient should be followed-up in the hospital or outpatient clinic or if there is a need for 
transferring to an advanced center. Additionally, early detection of the disease severity with a predictive calculation tool will provide the optimization of hospitalization duration, especially in countries with limited resources in terms of hospital beds and finances. Patients who are predicted not to need an ICU can be discharged from the hospital earlier. These reasons led to the construction of a COVID-19 severity score to predict severe illness that will require ICU follow-up based on the characteristics of the patients at admission to hospital and to develop a web-based calculation tool.

\section{Material And Method}

\section{Study Design and Participants}

This study was carried out in Ankara City Hospital, set apart as the reference pandemic center in Ankara with 3810 beds, of which 696 are intensive care beds. The ethical approval was obtained from Ankara City Hospital Ethical Committee 1. Verbal consent was obtained after the patients were informed that their medical records would only be used in medical studies by anonymizing their identity information. All patients older than 18 years who were hospitalized with the diagnosis of COVID-19 infection between March 15, 2020, and June 15, 2020, were included in the study. Patients who were still in hospital were excluded. The diagnosis was confirmed with polymerase chain reaction (PCR) for SARS-CoV-2 performed based on the protocol established by the World Health Organization (WHO) interim guideline [2]. Patients were monitored up to June 30,2020, the final date of follow-up. Data were analyzed between June 30, 2020 and July 5, 2020.

According to the WHO COVID-19 disease severity classification, disease without evidence of pneumonia or hypoxia was considered mild. Patients with signs and symptoms of pneumonia but no signs of severe pneumonia were considered moderate. Patients with pneumonia and one of the following; $>30$ breaths/min; severe respiratory distress; or Sp02 $<90 \%$ on room air were considered severe. Patients were considered critical if they had ARDS or other respiratory failure requiring mechanical ventilation, or septic shock, and/or organ failure requiring ICU follow-up [4]. The primary outcome was defined as severe illness that required ICU follow-up. The patients were classified as severe cases who required ICU followup and non-severe cases who did not require ICU follow-up based on disease severity. In the continuation of the text, the terms "severe patient" and "severe illness" are used to refer to the patient in need of the ICU and the disease that requires intensive care follow-up in the ICU.

\section{Collecting and Processing Data}

To collect data, a special form was created for COVID-19 patients, containing information of patients at the admission and follow-up. The parameters included in special patient forms were age, sex, epidemiological characteristics, comorbid diseases, duration and characteristic of symptoms and vital signs at admission. Laboratory and radiological tests were complete blood counts, serum biochemistry, C-reactive protein (CRP), procalcitonin (PCT), coagulation test, ferritin, D-dimer, troponin I, myoglobin, interleukin-6 (IL-6), Chest X-ray and thorax computed tomography (CT). The clinical outcomes including discharges, the requirement of ICU, death, and complications were recorded in patient forms. 
Data were collected prospectively. In case of patient death or discharge, all the missing laboratory records in patient files were completed from the hospital automation system and registered in an electronic recording system and uploaded collaboratively to an online database created specifically for COVID-19 patients. Data cut-off for the study was June 30, 2020. Data were recorded to the system by the physicians who followed-up the patients from different branches including infectious disease, internal medicine, respiratory disease, and anesthesiology and reanimation. After patient records were completed, the data was checked by two independent controllers who were infectious disease physicians. Patients with more than $30 \%$ missing data were not included in the study.

\section{Potential Predictive Parameters}

For the development of clinical severity score, patient data obtained on the day of hospitalization were used. Potential predictive parameters were determined from demographic, clinical, laboratory, and radiological characteristics, and their association with severe illness was investigated. A total of 68 predictors included in the severity score are shown Table 1 and 2.

\section{Statistical Analysis}

All analyses were performed using IBM SPSS V.21 software version. Descriptive statistics were presented as mean \pm standard deviation (SD) or medians plus with quartiles (IQR: 25th-75th percentile) for continuous variables and as frequency and percentages for categorical variables. Student $t$ test was used in the comparison of continuous variables for normally distributed data, and Mann Whitney $U$ test for non-normal distribution. Chi-square test was used for comparing categorical variables in parametric conditions. A p-value of less than 0.05 was considered statistically significant. Univariate and binary logistic regression were used to create a prediction model. Youden's $\mathrm{J}$ index was calculated with receiveroperator characteristic (ROC) curve analysis in order to evaluate cut-off points for continuous variables in order to simplify usage of the model.

Clinical parameters were assessed in terms of their association with severe illness requiring ICU follow-up by using ROC Curve Analysis. Then, Youden's J Index was calculated to decide the optimal breakpoint for each parameter. Univariate analysis was performed according to these cut-off points and parameters that were found to be statistically significant $(p<0.20)$ were included in multivariate analysis. To combine parameters that were found significant in univariate analysis, binary multivariate logistic regression analysis with a forward variable selection approach was carried out. After applying the logistic regression model, the estimated beta coefficients were used to assign score points. In the calculation tooldevelopment process, after logistic regression analysis, predicted probability was calculated for each patient by using the following formulas: probability $=\exp \left(\sum \beta \times \mathrm{X}\right) /\left[1+\exp \left(\sum \beta \times \mathrm{X}\right)\right]$. ROC analysis was performed for probability with a cut off point determined. The accuracy of the prediction model was tested by calculating the area under curve (AUC).

In the score-development phase, the beta coefficients in the model were multiplied by 10 and the value rounded off to the nearest integer. After calculating the probability for each patient, we used these 
probabilities as a combined diagnostic variable to assess the diagnostic performance of a new score by using ROC Curve Analysis. The performance of the scoring system was evaluated by using the cut-off value obtained from Youden's $\mathrm{J}$ Index for each patient in order to be grouped as cases needing ICU or not. The accuracy of the prediction model was tested by calculating AUC.

\section{Results}

\section{Characteristics of Study Cohort}

A total of 1022 patients with laboratory confirmed COVID-19 patients had been hospitalized during the study interval. Of the patients, 540 (52.8\%) were male and the median age was 47 (IQR 32-60) years. Baseline demographic, epidemiological and clinical characteristics of the patients are presented in Table 1. Of the patients, 90 (8.8\%) were severe and 27 (2.6\%) were critical upon admission based on WHO classification. Of the patients, 152 required ICU follow-up. The overall mortality rate was $4.9 \%(50)$.

To develop the severity score ACCSES, all 1022 patients hospitalized with PCR positive COVID-19 were included in the development cohort for variable selection and risk score calculation. As described herein, 68 variables were entered into the selection process. Demographic, epidemiological, clinical, laboratory and radiological parameters were each tested with univariate and multivariate analyses to investigate their relationship with serious diseases (Table 1-3).

Parameters that were evaluated as clinically significant and not correlated with each other, and those with less than $20 \%$ missing data were included in the univariate analysis. Parameters that did not differ between groups were not included in the scoring process. Among clinical symptoms and signs and laboratory and radiological findings, 20 parameters met the potential predictive factor condition for severe illness and were included in the disease severity score. These 20 parameters were age, sex, oxygen saturation, hypertension, chronic pulmonary disease, fever, neutrophil to lymphocyte ratio, monocyte, hemoglobin, platelet count, glomerular filtration rate, aspartate transaminase, alanine transaminase, lactate dehydrogenase (LDH), CRP, procalcitonin, ferritin, D-dimer, ilateral ground-glass opacity on CT. After the mentioned processes, univariate analysis was performed for all included 20 parameters. For the binary logistic regression, the forward method was used. Thus, the right parameters were chosen for the strongest model. Twenty parameters determined by univariate analysis were entered into the binary logistic regression analysis. The severity score was constructed with the 9 parameters calculated to be statistically significant in multivariate analysis.

\section{Independent High-Risk Factors for Severe Illness}

A further multivariate analysis with a forward variable selection approach showed final 9 parameters as the independent risk factor; these were sex (odds ratio [OR] 3.9, 95\% $\mathrm{Cl} 1.4-11.0$ ), oxygen saturation (OR 6.7, 95\% Cl 2.8-15.8), hemoglobin (OR 5.8, $95 \% \mathrm{Cl}$ 2.1-15.7), platelet (OR 3.4, 95\% Cl 1.4-10.5), glomerular filtration rate (GFR) (OR 6.5, 95\% Cl 2.5-16.7), aspartate transaminase (AST) (2.9, 95\% Cl 1.2-7.1), PCT (OR 2.6, 95\% Cl 1.1-6.3), ferritin (OR 3.0, 95\% Cl (1.2-7.1) and D-dimer (OR 4.7, 95\% Cl 1.8-12.2) (Table 3). 


\section{Construction of ACCSES Calculator and ACCSES Score}

The severity score, ACCSES, was created based on the coefficients obtained from the logistic model. The following formula was used for the logistic model to calculate the probability: probability $=\exp \left(\sum \beta x\right.$ $X) /\left[1+\exp \left(\sum \beta \times X\right)\right]$. Based on 9 independent high-risk factors for severe illness, a web-based calculation tool (ACCSES Calculation Tool) was constructed. The clinician can easily access the ACCSES Calculation tool using the online website at http: /www.achcovid19.com. ACCSES consists of 9 yes or no questions, and after answering the questions, the calculator estimates the result as needing ICU or not needing ICU. The online web-based ACCSES Calculation Tool is shown in Figure 1. The positive predictive value of the calculation tool was detected as $74,3 \%$, negative predictive value was $96,8 \%$. We have also developed ACCSES Manual Calculation Tool to be used by physicians according to preference, especially for those who do not prefer to use web-based techniques in the prediction of severe disease. Answering 9 questions with a yes or no choice is enough to estimate whether patients will need the ICU or not. In the manual tool, each of the 9 parameters has a point obtained from the beta coefficient for the prediction of severe patients. For each parameter, if the answer is yes, the patient gets the calculated point for the parameter, otherwise he/she will get 0 point. Whether the patient goes to the ICU or not is estimated based on the total score of the patient. The patients with a point above 63 are considered to need ICU follow-up. The ACCSES Manuel Calculation Tool is shown in Figure 2. We assessed the accuracy of ACCSES Calculation Tool and ACCSES Manual Calculation Tool by using AUC, which showed good performance (Figure 3.).

\section{Discussion}

Besides being a new and unknown disease, the increasing number of COVID-19 patients in a short time caused heavy burden and stress on healthcare providers [5]. The lack of human resources in the field of health, the shortage of hospital bed capacities and limited financial resources have made it necessary to select severe patients who may have a serious course to be provided health services first. Therefore, we developed a simple, accessible, easy to use calculation tool that makes physician's decisions on the management of COVID-19 patients easier and provides ways of recognizing severe illness requiring ICU by using available and accessible values of patients on admission. The physician who first evaluated patient in primary care hospitals or in emergency department can decide where the patient should be followed-up as an inpatient or as an outpatient. The calculation tool also helps the physicians who are working in neighboring provinces and districts to decide whether patients will be transferred to tertiary hospitals or not.

The strongest calculation tool was constructed with two clinical (sex and oxygen saturation) and seven laboratory parameters (hemoglobin level, platelet count, GFR, AST and PCT level, ferritin and D-dimer) and it exhibited very strong distinctive power in the prediction of severe illness upon admission. It can identify a small proportion of cases whose illness will progress with a positive predictive value of $74.3 \%$ and a very high sensitivity of $92 \%$. To our knowledge, it has the strongest discrimination properties for predicting severe cases of COVID-19 in the literature. These parameters included in ACCSES actually 
reflect the multi-organ functions and represent almost the whole human body including pulmonary, renal, hepatic and hematological system. Additionally, it contains inflammatory indicators. Therefore, it provides the opportunity to make an integrated decision about the patient and results in accurate estimation.

There is a number of studies investigating severity [6-12] and mortality [8,13-16] predictors in COVID-19 infection. Some of the studies reported that they developed scores or models for the prediction of disease severity [6-12]. Shi et al. defined a host risk score consisting of three parameters (age, sex, hypertension) and reported an increase in severe infection risk with the increasing score point (from 0 to 3) [12]. However, this scoring system does not determine a specific risk for each patient. Liang $W$ et al. reported that they developed and validated a clinical risk score named as COVID-GRAM with ten parameters (chest radiographic abnormality, hemoptysis, dyspnea, age, unconsciousness, number of comorbidities, cancer histories, neutrophil-to-lymphocyte ratio, $\mathrm{LDH}$, and direct bilirubin) [6]. The mean AUC was reported as $0.88(95 \% \mathrm{Cl}, 0.85-0.91)$ in the development group and as $0.88(95 \% \mathrm{Cl}, 0.84-0.93)$ in the validation group. Zhou et al. also reported that they developed a nomogram to predict severe patients. Seven clinical variables (body temperature at admission, oxygen saturation, cough, dyspnea, hypertension, cardiovascular disease, chronic liver disease, and chronic kidney disease on admission) were included in the nomogram. [7]. The nomogram was reported to have a good discrimination with an area under the curve of 0.862 , C-index of 0.863 (95\% confidence interval, $0.801-0.925$ ). National Early Warning Systems 2 (NEWS2), an early warning systems used for prediction of cardiac arrest risk, unexpected intensive care unit admission or death, was recommended by the National Institute of Clinical Excellence (NICE) for the prediction of critical patients in COVID-19. However, Sze S et al. reported that NEWS2 score does not predict disease severity, no difference was detected between the groups in terms of NEW2 score [17].

Levy et al. established some clinical predictive tools consisting of 6 factors (blood urea nitrogen, patient age, absolute neutrophil count, red cell distribution width, oxygen saturation, and serum sodium) which resulted in an AUC of 0.86 to predict 7-day mortality. In another study developing models, clinical (age, hypertension, and coronary arterial disease) and laboratory (age, high sensitive CRP, oxygen saturation, neutrophil and lymphocyte count, D-dimer, AST and GFR) mortality-prediction models were developed. The AUC values were reported as $0.83(95 \% \mathrm{Cl} 0.68-0.93)$ and $0.88(95 \% \mathrm{Cl} 0.75-0.96)$ for the clinical model and laboratory model, respectively [15].

Age was reported as a predictor for severe COVID-19 infection [15,16,11,18-21]. In our study, severe cases were detected to be older than non-severe cases, and age was included in the score development process, however, it could not enter the final calculation tool. The association between comorbid diseases and the development of severe infection was indicated in previous studies $[22,15,11,16,12,13]$ Some were the severity and mortality risk scores [15,11]. Similarly, although we found that comorbid diseases were higher in severe cases than non-severe patients, and severe patients had a higher number of comorbidities than non-severe cases, comorbidities were not identified as optimal predictors during the development process of the calculation tool. 
Whether sex difference creates a predisposition to COVID-19 infection or severe form of the disease was discussed in a few studies [23-25]. It was emphasized that higher smoking rates or higher angiotensinconverting enzyme-2 (ACE2) expression in men may be a predisposing factor [23]. A current study from the United Kingdom (UK) reported that men had a higher risk for COVID-19 infection than women in a large population of 17,278,392 adults (fully adjusted hazard ratio [HR] 1.59, 1.53-1.65) [23]. In our study, male sex was found as a predictor for severe outcome and entered the final version of the score. Similar to our study, Shi et al. reported male sex as an independent factor (OR 3.68, 95\% $\mathrm{Cl} 1.75-7.75$ ) for severe disease and included it in the host risk score [15].

Our study has some limitations. Firstly, although the study population is enough for the development of severity score, it was not sufficient to validate the results of study population. Maybe further studies with large number can validate ACCSES Calculation Tool in a different study population. Secondly, we can

perform the study in single center. The more extensive, multicenter and large sample size studies will be better to represent the whole population.

\section{Conclusion}

We developed a calculation tool for the prediction of severe illness requiring ICU with good distinctive power. Using the ACCESS calculation tool provides some benefits to the clinician by using available clinical and laboratory parameters upon admission. The clinician can decide more easily where the patient should be followed, in the hospital or outside (at home, in the isolation institute or nursing home). Additionally, patients who have severe illness or have a potential for worsening in the following days can be transferred earlier to the appropriate clinic (ICU if necessary) to begin treatment. Finally, the optimal use of hospital beds can be provided by preventing unnecessarily long hospitalizations in those who were predicted low-risk for the severe outcome, especially in countries that have limited sources in terms of hospital beds or financial capacity.

\section{Declarations}

\section{Author Contributions:}

All authors have made substantial contributions to the study. Study concept and design (BK and RG), acquisition of the data (IH, AA, AKK, HB, IA, EA, DE, SI, AA, AK, AAS, MA, SK, IOT, EA, BO, MNM, YAB, EMS, $D G, B D, S G, E G, R B, O A, D A, O I, T B$ ), analysis and interpretation of the data (BK, RG and IH), drafting of the manuscript (BK and $R G$ ), critical revision of the manuscript for important intellectual content (RG, $F E$ ), statistical expertise, and acquisition of funding (BK, RG). All authors contributed to the review and read and approved the final manuscript

\section{Funding Sources}

The authors declare that they have no conflict of interest. 


\section{Conflict of interest}

The authors have no conflicts of interest to declare.

\section{Ethical approval}

The research was conducted ethically in accordance with the World Medical Association Declaration of Helsinki. The study was approved by the Turkish Ministry of Health and Ankara City Hospital, Ethical Committee 1.

\section{Statement of human and animal rights}

This article does not contain any study with animals.

\section{Informed consent}

In this study, verbal consent was obtained from the patients

\section{Availability of data and materials}

The data analyzed in this study can be provided from the corresponding author upon reasonable request.

\section{Abbreviations}

COVID-19: Coronavirus Disease-2019

SARS-CoV-2: Severe Acute Respiratory Syndrome Coronavirus-2

CT: Computer tomography

WHO: World Health Organization

ARDS: Acute respiratory distress syndrome

PCR: Polymerase chain reaction assay

ICU: Intensive care unit

SD: Standard deviation

IQR: Inter-quartile range

OR: Odds ratio

Cl: Confidence interval

ACCSES: Ankara City Hospital COVID-19 Severity Score 


\section{References}

1. World Health Organization. Coronavirus disease (COVID-19) Dashboard. https://covid19.who.int/. Accessed 10 July 2020.

2. World Health Organization. Clinical management of COVID-19. interim guidance, 27 May 2020. https://www.who.int/publications-detail/clinical-management-of-covid-19. Licence CC BY-NC-SA 3.0 IGO. Accessed 10 July 2020.

3. Wu Z, McGoogan JM. Characteristics of and important lessons from the Coronavirus Disease 2019 (COVID-19) outbreak in China: Summary of a report of 72314 cases from the Chinese Center for Disease Control and Prevention. JAMA. 2020;10.1001/jama.2020.2648.

4. World Health Organization. Coronavirus disease (COVID-19) technical guidance: laboratory testing for 2019-nCoV in humans. https://www.who.int/emergencies/diseases/novel-coronavirus2019/technical -guidance/laboratory-guidance. Accessed 08 July 2020.

5. Koçak Tufan Z, Kayaaslan B. Crushing the curve, the role of national and international institutions and policy makers in COVID-19 pandemic. Turk J Med Sci. 2020;50(SI-1):495-508.

6. Liang W, Liang H, Ou L, Chen B, Chen A, Li C, et al. Development and validation of a clinical risk score to predict the occurrence of critical illness in hospitalized patients with COVID-19. JAMA Intern Med. 2020;e202033.

7. Zhou Y, He Y, Yang H, Yu H, Wang T, Chen Z, et al. Development and validation a nomogram for predicting the risk of severe COVID-19: A multi-center study in Sichuan, China. PLoS One. 2020;15(5):e0233328.

8. Levy TJ, Richardson S, Coppa K, Barnaby DP, McGinn T, Becker LB, et al. Development and validation of a survival calculator for hospitalized patients with COVID-19. Preprint. medRxiv. 2020;2020.04.22.20075416.

9. Verity R, Okell LC, Dorigatti I, Winskill P, Whittaker C, Imai N, et al. Estimates of the severity of coronavirus disease 2019: a model-based analysis [published correction appears in Lancet Infect Dis. 2020 Apr 15;] [published correction appears in Lancet Infect Dis. 2020 May 4;]. Lancet Infect Dis. 2020;20(6):669-677.

10. Singh K, Valley TS, Tang S, Li BY, Kamran F, Sjoding MW, et al. Validating a widely implemented deterioration index model among hospitalized COVID-19 patients. Preprint. medRxiv. 2020;2020.04.24.20079012.

11. Ji D, Zhang D, Xu J, Chen Z, Yang T, Zhao P, et al. Prediction for progression risk in patients with COVID-19 pneumonia: the CALL Score. Clin Infect Dis. 2020;ciaa414.

12. Shi Y, Yu X, Zhao H, Wang H, Zhao R, Sheng J. Host susceptibility to severe COVID-19 and establishment of a host risk score: findings of 487 cases outside Wuhan. Crit Care. 2020;24(1):108.

13. Du R-H, Liang L-R, Yang C-Q, Wang W, Cao T-Z, Li M, et al. Predictors of mortality for patients with COVID-19 pneumonia caused by SARS-CoV-2: a prospective cohort study. Eur Respir J. 2020;55(5):2000524. 
14. Mizumoto K, Chowell G. Estimating risk for death from Coronavirus disease, China, January-February 2020. Emerg Infect Dis. 2020;26(6):1251-1256.

15. Wang K, Zuo P, Liu Y, Zhang M, Zhao X, Xie S, et al. Clinical and laboratory predictors of in-hospital mortality in patients with COVID-19: a cohort study in Wuhan, China. Clin Infect Dis. 2020;ciaa538.

16. Ruan Q, Yang K, Wang W, Jiang L, Song J. Clinical predictors of mortality due to COVID-19 based on an analysis of data of 150 patients from Wuhan, China. Intensive Care Med. 2020;46(5):846-848.

17. Sze S, Pan D, Williams CM, Wong N, Sahota A, Bell D, et al. Letter to the Editor: Variability but not admission or trends in NEWS2 score predicts clinical outcome in elderly hospitalised patients with COVID-19. J Infect. 2020;S0163-4453(20)30341-8.

18. Wang D, Hu B, Hu C, Zhu F, Liu X, Zhang J, et al. Clinical characteristics of 138 hospitalized patients with 2019 novel coronavirus-infected pneumonia in Wuhan, China. Jama, 2020, 323.11: 1061-1069

19. Wang B, Li R, Lu Z, Huang Y. Does comorbidity increase the risk of patients with COVID-19: evidence from meta-analysis. Aging (Albany NY). 2020;12(7):6049-6057.

20. Yadaw AS, Li YC, Bose S, lyengar R, Bunyavanich S, Pandey G. Clinical predictors of COVID-19 mortality. Preprint. medRxiv. 2020;2020.05.19.20103036.

21. Zhou F, Yu T, Du R, Fan G, Liu Y, Liu Z, et al. Clinical course and risk factors for mortality of adult inpatients with COVID-19 in Wuhan, China: a retrospective cohort study [published correction appears in Lancet. 2020 Mar 28;395(10229):1038] [published correction appears in Lancet. 2020 Mar 28;395(10229):1038]. Lancet. 2020;395(10229):1054-1062.

22. Hu Y, Sun J, Dai Z, Deng H, Li X, Huang Q, et al. Prevalence and severity of corona virus disease 2019 (COVID-19): A systematic review and meta-analysis. J Clin Virol. 2020;127:104371.

23. Cai H. Sex difference and smoking predisposition in patients with COVID-19 [published correction appears in Lancet Respir Med. 2020 Apr;8(4):e26]. Lancet Respir Med. 2020;8(4):e20.

24. Williamson EJ, Walker AJ, Bhaskaran K, Bacon S, Bates C, Morton CE, et al. OpenSAFELY: factors associated with COVID-19 death in 17 million patients. Nature. 2020;10.1038/s41586-020-2521-4.

25. Zhang JJ, Dong X, Cao YY, Yuan YD, Yang YB, Yan YQ, et al. Clinical characteristics of 140 patients infected with SARS-CoV-2 in Wuhan, China. Allergy. 2020;75(7):1730-1741.

\section{Tables}

TABLE 1. Baseline Demographic and Clinical Characteristics of Patients at Admission (41 parameters) 


\begin{tabular}{|c|c|c|c|c|}
\hline Characteristics & $\begin{array}{l}\text { All } \\
\text { patients } \\
(n=1022)\end{array}$ & $\begin{array}{l}\text { Severe patients } \\
(n=152)\end{array}$ & $\begin{array}{l}\text { Non-severe } \\
\text { patients } \\
(n=870)\end{array}$ & $\begin{array}{l}P \\
\text { value }\end{array}$ \\
\hline Age (median, IQR*) & $\begin{array}{l}47(32- \\
60)\end{array}$ & $67(57-76)$ & $41(31-54)$ & $<0.001$ \\
\hline Sex, male & $\begin{array}{l}540 \\
(52.8)\end{array}$ & $94(61.8)$ & $446(51.3)$ & 0.016 \\
\hline Smoking status $(n=587)$ & & & & 0.001 \\
\hline Never smokes & $\begin{array}{l}423 \\
(72.1)\end{array}$ & $35(53.8)$ & $388(74.3)$ & \\
\hline Active or ex-smoker & $\begin{array}{l}164 \\
(19,9)\end{array}$ & $30(46.2)$ & $144(25.7)$ & \\
\hline $\begin{array}{l}\text { Exposure to COVID-19 patients in the past } \\
14 \text { days }(n=805)\end{array}$ & $\begin{array}{l}519 \\
(64.5)\end{array}$ & $40(48.2)$ & $479(66.3)$ & 0.001 \\
\hline $\begin{array}{l}\text { Travel history to high-risk area in the past } \\
14 \text { days }(\mathrm{n}=1017)\end{array}$ & $100(9.8)$ & $21(13.8)$ & $79(9.1)$ & 0.074 \\
\hline Any comorbidity & $\begin{array}{l}405 \\
(39.6)\end{array}$ & $112(73.7)$ & 293 (33.7) & $<0.001$ \\
\hline Number of comorbidities & & & & $<0.001$ \\
\hline$\leq 1$ & $\begin{array}{l}791 \\
(77.4)\end{array}$ & $74(48.7)$ & $717(82.4)$ & \\
\hline$\geq 2$ & $\begin{array}{l}231 \\
(22.6)\end{array}$ & $78(51.3)$ & $153(17.6)$ & \\
\hline Diabetes & $\begin{array}{l}129 \\
(12.6)\end{array}$ & $39(25.7)$ & $90(10.3)$ & $<0.001$ \\
\hline Hypertension & $\begin{array}{l}218 \\
(21.3)\end{array}$ & $67(44.1)$ & $151(17.4)$ & $<0.001$ \\
\hline Coronary artery disease & $73(7.1)$ & $28(18.4)$ & $45(5.2)$ & $<0.001$ \\
\hline Other cardiac disease & $24(2.3)$ & $9(5.9)$ & $15(1.7)$ & 0.002 \\
\hline Chronic pulmonary disease & $93(9.1)$ & $30(19.7)$ & $63(27)$ & $<0.001$ \\
\hline Neurological diseases & $24(2.3)$ & $14(9.2)$ & $10(1.1)$ & $<0.001$ \\
\hline Chronic renal disease & $16(1.6)$ & $7(4.6)$ & $9(1.0)$ & 0.001 \\
\hline Malignity & $31(3.0)$ & $14(9.2)$ & $17(2.0)$ & $<0.001$ \\
\hline Pregnancy $(n=482)$ & $26(6.1)$ & 0 & $26(6.1)$ & 0.53 \\
\hline Thyroid disease & $32(3.1)$ & $8(5.3)$ & $24(2.8)$ & 0.10 \\
\hline
\end{tabular}


Data are presented as $\mathrm{n}(\%)$ unless noted otherwise.

Data are presented for 1022 patients unless noted otherwise.

Severe patients were defined as those requiring intensive care unit follow-up.

*IQR: Interquartile range (25\% and $75 \%)$

TABLE 1. Baseline Demographic and Clinical Characteristics of Patients at Admission (41 parameters) (continued) 


\section{Characteristics}

$\begin{array}{ll}\text { All patients } & \text { Severe } \\ (n=1022) & \begin{array}{l}\text { patients } \\ (n=152)\end{array}\end{array}$

Non-severe

patients

$(n=870)$

\section{Symptoms}

Any symptom

Time between symptom onset and

hospitalization $(n=743)$ (median, IQR*)

Fever

Cough

Expectoration

Dyspnea

Sore throat

Runny nose

Nausea and vomiting

Diarrhea

Fatigue

Loss of appetite

Arthralgia and myalgia

Headache

Chill

Loss of smell

Loss of taste

Vital parameters

Body temperature, $>37.8 \otimes C(n=1022)$

Respiratory rate, $/ \min (\mathrm{n}=883)$

SBP $>140 \mathrm{mmHg}$ or DBP $>90 \mathrm{mmHg}$ $(\mathrm{n}=926)$ **

Heart rate $>100 / \mathrm{min}$
$869(85.0)$

$3(2-7)$

$377(36.9) \quad 76(50.0)$

$524(51.3) \quad 85(55.9)$

$39(3.8)$

$257(25.1)$

$176(17.2)$

$9(5.9)$

$36(3.5)$

$60(5.9)$

$65(6.4)$

207 (20.3)

$42(4.1)$

$217(21.2) \quad 16(10.5)$

$118(11.5)$

$10(6.6)$

$7(4.6)$

$1(0.7)$

$4(2.6)$

$59(5.8)$

177 (17.3)

$120(13.8)$

$57(37.5) \quad<0.001$

$21(14-45), \quad 20$ (18-22) IQR 19-22

$68(7.3)$

$53(5.2)$

$39(26.7)$

$728(83.9) \quad 0.001$

$3(2-6)$

0.38

$301(34.6)<0.001$

439 (50.5) $\quad 0.21$

$23(2.6) \quad<0.001$

$169(19.4)<0.001$

$167(19.2) \quad<0.001$

$33(3.8) \quad 0.26$

$49(5.6)$

0.43

$59(6.8)$

0.19

$177(20.3) \quad 0.87$

28 (3.2) $\quad 0.001$

$201(23.1) \quad 0.001$

$108(12.4) \quad 0.04$

$54(6.2) \quad 0.44$

$55(6.3) \quad 0.005$

$55(6.3) \quad 0.07$

Page 15/20 
$\leq 1$ point

$821(94.0)$

80 (9.7)

$741(90.3)$

$\geq 2$ point

$52(6.0)$

$49(94.2)$

$3(5.8)$

Data are presented as $\mathrm{n}(\%)$ unless noted otherwise.

Data are presented for 1022 patients unless noted otherwise.

Severe patients were defined as those requiring intensive care unit follow-up.

*IQR: Interquartile range (25\% and $75 \%)$

**SBP: Systolic blood pressure, DBP: diastolic blood pressure

*** SOFA: Sequential organ failure assessment 
TABLE 2. Baseline Laboratory and Radiological Characteristics of Patients at Admission (27 parameters)

\section{Variable}
All patients
$(n=1022)$

\section{Laboratory values}

White blood cell, $\times 10^{9} / \mathrm{L}$

Neutrophil count, $\times 10^{9} / \mathrm{L}$

Lymphocyte count, $x 10^{9} / \mathrm{L}$

Neutrophil to lymphocyte ratio

Monocyte count, $\times 10^{9} / \mathrm{L}$

Hemoglobin, g/L

Platelet count, $\times 10^{9} / \mathrm{L}$

C-reactive protein

Procalcitonin, $\mathrm{pg} / \mathrm{mL}$

Lactate dehydrogenase, U/L

Aspartate transaminase, $\mathrm{U} / \mathrm{L}$

Alanine transaminase, $\mathrm{U} / \mathrm{L}$

Glomerular filtration rate,

$\mathrm{ml} / \mathrm{min} / 1.73 \mathrm{~m} 2$

Creatinine kinase, $\mu / L$

Albumin, $\mathrm{g} / \mathrm{L}$

D-dimer, $\mathrm{mg} / \mathrm{L}$

Ferritin, $\mu \mathrm{g} / \mathrm{L}$

Prothrombin time (PT), sec.

24 (17-36)

$26(18-40)$

Non-severe
patients
$(n=870)$

Median (IQR)* Median (IQR)

$5500(4330-\quad 6190(4230-9600)$

7430)

3320 (2490- $\quad 4500$ (3080-7080)

4600)

$1240(900-\quad 840(600-1110)$

1760)

$2.5(1.6-4.2)$

$5.0(3.2-9.7)$

$360(270-480)$

$320(210-480)$

$13.7(12.5-$

14.9)

$213(172-262)$

0.009 (0.003- $\quad 0.09(0.03-0.15)$

$0.33)$

$12.7(11.2-14.2)$

$200(152-259)$

$0.03(0.03-$

$0.07)$

$0.12(0.06-.030)$

Median (IQR)

7120) 4260) 1820) 15.0) $0.02)$ $0.05)$
$\mathbf{P}$ value

$5410(4350-\quad<0.001$

$3220(2410-\quad<0.001$

$1310(980-\quad<0.001$

$2.3(1.5-3.7) \quad<0.001$

$360(270-480) \quad<0.001$

$13.8(12.6-\quad<0.001$

$215(176-260) \quad<0.001$

$0.006(0.003-<0.001$

$0.030(0-0.03-<0.001$

218 (187-284) 341 (252-456)

$211(184-261)<0.001$

39 (19-48)

$22(17-32)$

$<0.001$

$27(19-48)$

26 (18-39)

$<0.001$

101 (83-114) 83 (60-96)

$103(87-116)<0.001$

89 (59-147) 142 (73-386)

87 (58-139)

$<0.001$

$44(21-63)$

$38.1(25-42)$

$45(42-47)$

$<0.001$

$0.4(0.27-\quad 1.0(0.6-2.2)$

$0.4(0.2-0.6)$

$<0.001$

$0.81)$

$117(44-280)$

419 (174-694)

98 (38-218)

$<0.001$

$12.1(11.7-\quad 13.0(12.0-14.0)$

12.8)
12.0 (11.6-

12.6) $<0.001$ 


\begin{tabular}{|c|c|c|c|c|}
\hline $\begin{array}{l}\text { Activated partial thromboplastin } \\
\text { time, sec. }\end{array}$ & $25.1(23-26)$ & $25.8(23.0-27.8)$ & $\begin{array}{l}25.0(23.5- \\
26.5)\end{array}$ & $<0.001$ \\
\hline INR & $1.03(1.0-1.1)$ & $1.1(1.0-1.2)$ & $1.02(0.9-1.0)$ & $<0.001$ \\
\hline Fibrinogen, $\mathrm{g} / \mathrm{L}$ & $3.2(2.6-4.1)$ & $4.5(3.5-5.7)$ & $3.1(2.5-3.9)$ & $<0.001$ \\
\hline Interleukin 6 (IL-6), pg/mL & $24.5(9-56)$ & $43(26-118)$ & $15(7-35)$ & $<0.001$ \\
\hline Troponin, ng/L & $2.5(2.5-6)$ & $10(5.0-23)$ & $2.5(2.5-5.0)$ & $<0.001$ \\
\hline Myoglobin, mg/L & $42(27-71)$ & $97(55-185)$ & $38(25-60)$ & $<0.001$ \\
\hline \multicolumn{5}{|l|}{ CT** abnormality $(\mathrm{n}=956)$} \\
\hline Any finding & 757 (79.2) & $140(95.9)$ & $617(76.7)$ & $<0.001$ \\
\hline Consolidation & $199(20.8)$ & $28(19.2)$ & $78(9.6)$ & $<0.001$ \\
\hline Unilateral ground-glass opacity & $171(17.9)$ & $9(5.5)$ & $162(18.6)$ & $<0.001$ \\
\hline Bilateral ground-glass opacity & $487(50.9)$ & $117(77)$ & 370 (42.5) & $<0.001$ \\
\hline \multicolumn{5}{|c|}{ Data are presented for 1022 patients unless noted otherwise. } \\
\hline \multicolumn{5}{|c|}{ Severe patients were defined as those requiring intensive care unit follow-up. } \\
\hline \multicolumn{5}{|c|}{ *IQR: Interquartile range (25\% and $75 \%)$} \\
\hline \multicolumn{5}{|l|}{${ }^{\star \star} \mathrm{CT}$ : Computed Tomography } \\
\hline
\end{tabular}

Table 3. Multivariate Logistic Regression Analysis for Prediction of Severe COVID-19 Infection Requiring Intensive Care Unit Follow-up 


\begin{tabular}{|llll|}
\hline Variables & Beta coefficient & $\begin{array}{l}\text { Odds ratio } \\
(95 \% \mathrm{Cl} \text { ) }\end{array}$ & P value \\
\hline Sex, male & 1,38 & $3.91(1.43-11.04)$ & 0.008 \\
\hline Saturation, $<94 \%$ & 1,91 & $6.75(2.88-15.85)$ & $<0.001$ \\
\hline Hemoglobin, $<13.0 \mathrm{~g} / \mathrm{L}$ & 1,77 & $5.85(2.17-15.77)$ & $<0.001$ \\
\hline Platelet count, $<135 \times 10^{9} / \mathrm{L}$ & 1,24 & $3.46(1.14-10.50)$ & 0.028 \\
\hline Glomerular filtration rate, $<98 \mathrm{ml} / \mathrm{min} / 1.73 \mathrm{~m} 2$ & 1,88 & $6.52(2.54-16.70)$ & $<0.001$ \\
\hline Aspartate transaminase, $<29 \mathrm{U} / \mathrm{L}$ & 1,08 & $2.93(1.19-7.18)$ & 0.02 \\
\hline Procalcitonin, $>0.085 \mathrm{pg} / \mathrm{mL}$ & 0,98 & $2.66(1.11-6.36)$ & 0.03 \\
\hline Ferritin, $<228 \mu \mathrm{g} / \mathrm{L}$ & 1,11 & $3.02(1.29-7.06)$ & 0.01 \\
\hline D-dimer, $>0.53 \mathrm{mg} / \mathrm{L}$ & 1,55 & $4.72(1.82-12.20)$ & 0.001 \\
\hline * Confidence $\mathrm{Interval}$ & & & \\
\hline
\end{tabular}

\section{Figures}

\begin{tabular}{|c|c|c|c|}
\hline \multicolumn{4}{|c|}{ Figure 2. Ankara City Hospital COVID-19 Severity Score (ACCSES) Manuel Calculation Tool for Prediction } \\
\hline Variables & Criterion & Value* & Enter value or 0 \\
\hline Sex (male) & Male & 14 & \\
\hline Saturation & 94 and below & 19 & \\
\hline Hemoglobin & 13,0 and below & 18 & \\
\hline Platelet & 135 and below & 12 & \\
\hline Glomerular filtration rate (GFR) & 98 and below & 19 & \\
\hline Aspartate transaminase (AST) & 29 and above & 11 & \\
\hline Procalcitonin (PCT) & 0.085 and above & 10 & \\
\hline Ferritin & 228 and above & 11 & \\
\hline \multirow[t]{2}{*}{ D-dimer } & 0,53 an above & 16 & \\
\hline & & & Total value $=$ \\
\hline
\end{tabular}

\begin{tabular}{|l|l|}
\hline PREDICTION \\
\hline Total value $>63$ & Need for ICU \\
\hline Total value $\leq 63$ & No need for ICU \\
\hline
\end{tabular}


Figure 1

Online Web-based ACCSES Calculator for the Prediction of Severe Cases Requiring ICU Follow-up

Figure 3. ROC* Curves and Characteristics of ACCSES Calculation Tool and Manuel Calculation

Tool

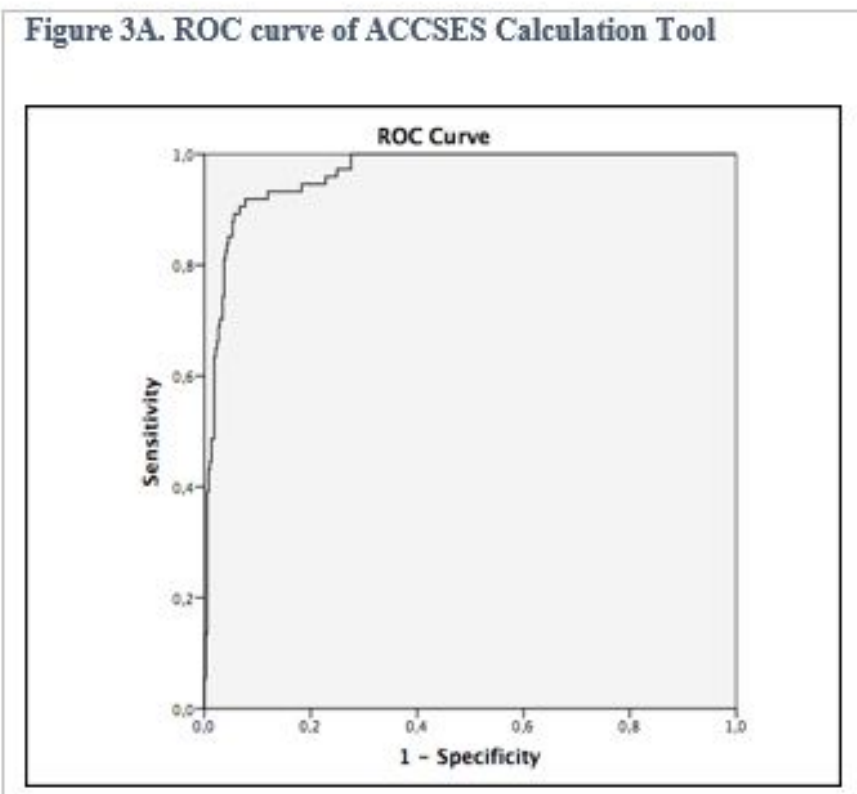

Figure 3B. ROC curve of ACCSES Manual Calculation Tool

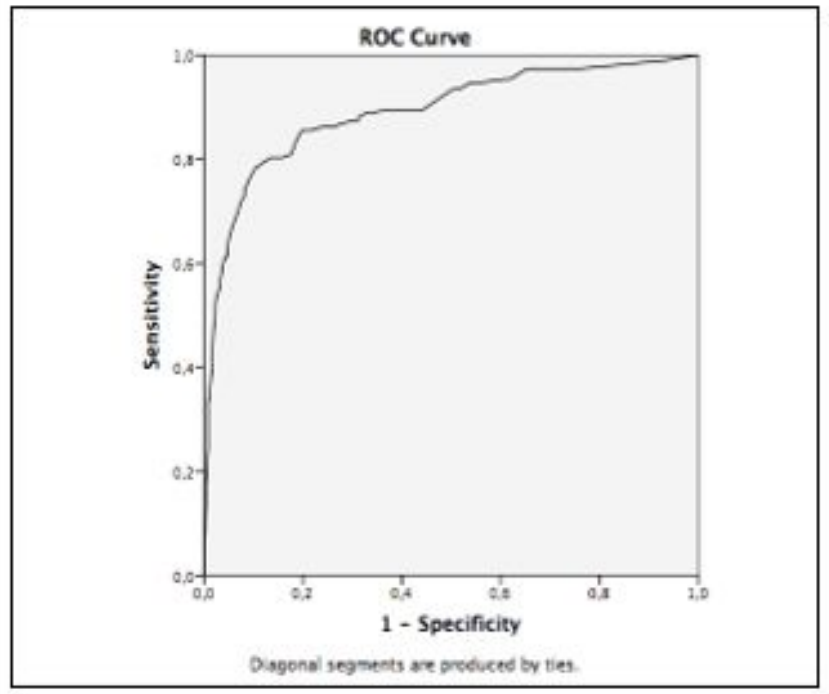

\begin{tabular}{|l|c|c|}
\hline & ACCSES Calculation Tool & ACCSES Manual Calculation Tool \\
\hline AUC $(95 \%$ CI) & $0.96(0.95-0.98)$ & $0.89(0.86-0.92)$ \\
\hline Threshold & 0,2469602 & 63,5 \\
\hline Sensitivity, $\%$ & 92 & 98 \\
\hline Specificity, $\%$ & 92 & 40.4 \\
\hline Positive predictive value, $\%$ & 74.3 & 98.6 \\
\hline Negative predictive value, $\%$ & 96.8 & 90 \\
\hline
\end{tabular}

*ROC: Receiver-operator characteristic

** AUC: Area under curve

\section{Figure 2}

Ankara City Hospital COVID-19 Severity Score (ACCSES) For Prediction of Severe COVID-19 Infection Requiring ICU Follow-up 